\title{
Performance Improvements in Raman Distributed Temperature Sensor
}

\author{
Toru FUKUZAWA, Hideo SHIDA, Kazushi OISHI, \\ Nobuaki TAKEUCHI, and Shoji ADACHI* \\ Yokogawa Electric Corporation, 2-9-32 Naka-cho, Musashino-shi, Tokyo 180-8750, Japan \\ ${ }^{*}$ Corresponding author: Shoji ADACHI \\ E-mail: Shouji.Adachi@jp.yokogawa.com
}

\begin{abstract}
The fiber optic distributed temperature sensor (DTS) is one of the most outstanding means to measure temperature distribution along an optical fiber. In this paper, we propose a novel calibration technique to measure the temperature highly accurately over a wide range of temperatures. We also propose an improved double-ended configuration that is insusceptible to the differential loss change in the fiber and suitable for the field use. Then, we developed an interrogator that had high robustness in harsh environments.
\end{abstract}

Keywords: Raman scattering, DTS, distributed temperature sensor, optical fiber sensor

Citation: Toru FUKUZAWA, Hideo SHIDA, Kazushi OISHI, Nobuaki TAKEUCHI, and Shoji ADACHI, "Performance Improvements in Raman Distributed Temperature Sensor," Photonic Sensors, DOI: 10.1007/s13320-013-0128-1.

\section{Introduction}

Fiber optic distributed sensors, which are a unique technology with attractive applications, are capable of long-distance measurement, explosion proof, resistant corrosion, free maintenance, light weight, and extreme durability [1].

In recent years, the Raman distributed temperature sensor (DTS) has been successfully adopted in the oil \& gas fields for monitoring the progress of the steam-assisted extraction process, inflow profile, injectivity profile of the injection well, etc. For these applications, the Raman DTS must be robust under 1) harsh environments (no electric power, wide operation temperature range, and corrosive gases), 2) high temperature $\left(\sim 300^{\circ} \mathrm{C}\right)$ measurement, and 3) exposure to hydrogen gases, resulting in the temperature measurement error.

In this paper, we propose a novel calibration technique to perform highly accurate measurement over a wide range of temperatures. Also, we propose an improved double-ended configuration that is insusceptible to the differential loss change in the fiber. Lastly, we introduce an interrogator that has high robustness in harsh environments.

\section{Principle}

Raman scattering occurs due to the interaction between light and optical phonons present in a continuum medium. In such interaction, the medium can lose energy (anti-Stokes) or acquire energy (Stokes) by means of scattering. Molecular vibrational states - the phonons - obey the BoseEinstein statistics distribution, which is a function of the temperature. At the room temperature, most molecules are at the lower energy state, so anti-Stokes scattering is even rarer than Stokes scattering. Stokes and anti-Stokes intensities $\left(I_{\mathrm{st}}\right.$,

Received: 16 July 2013 / Revised version: 28 August 2013

(C) The Author(s) 2013. This article is published with open access at Springerlink.com 
$\left.I_{\text {as }}\right)$ are given by

$$
\begin{aligned}
& I_{\text {st }} \propto\left(v_{0}-\Delta v\right)^{4} \cdot(1+n(\Delta v, T)) \\
& I_{\text {as }} \propto\left(v_{0}+\Delta v\right)^{4} \cdot n(\Delta v, T)
\end{aligned}
$$

where $v_{0}$ is the wave number of incident light, $\Delta v$ is the Raman shift wave number, and $n$ is the number of photons as expressed by

$$
n(\Delta v, T)=\frac{1}{\exp (h c \Delta v / k T)-1}
$$

where $h$ is Plank's constant, k is Bolzman's constant, and $c$ is the speed of light. The Raman shift wave number is determined by the molecular structure of the medium. The Raman spectrum has a peak around at from $400 \mathrm{~cm}^{-1}$ to $500 \mathrm{~cm}^{-1}$ in the silica optical fiber.

The ratio of anti-Stokes to Stokes intensities can be expressed as

$$
R(T)=\frac{I_{\text {as }}}{I_{\text {st }}}=\frac{\left(v_{0}+\Delta v\right)^{4}}{\left(v_{0}-\Delta v\right)^{4}} \cdot \exp \left(-\frac{h c \Delta v}{k T}\right) .
$$

Using the temperature of the built-in fiber (for temperature reference) $T_{0}$,

$$
R(T) / R\left(T_{0}\right)=\exp \left(-\frac{h c \Delta v}{k T}\right) / \exp \left(-\frac{h c \Delta v}{k T_{0}}\right) .
$$

Taking the logarithm of both sides,

$$
\begin{aligned}
& \ln \frac{R(T)}{R\left(T_{0}\right)}=-\frac{h c \Delta v}{k T}+\frac{h c \Delta v}{k T_{0}} \\
& \frac{1}{T}=\frac{1}{T_{0}}-\frac{k}{h c \Delta v} \ln \frac{R(T)}{R\left(T_{0}\right)} .
\end{aligned}
$$

Then,

$$
T=\frac{h c \Delta v}{k} \cdot\left[\frac{1}{-\ln R(T)+\ln R\left(T_{0}\right)+\frac{h c \Delta v}{k} \cdot \frac{1}{T_{0}}}\right] .
$$

The local temperature $T$ can be determined by knowing $T_{0}, R\left(T_{0}\right), R(T)$, and $\Delta v$ [2]. Here, we assume that the deferential loss (attenuation difference at the Stokes and anti-Stokes wavelengths) is corrected beforehand, and the sensing fiber has the same characteristics as the built-in fiber. Moreover, we assume that the optical connector does not have the differential loss.

\section{Error factors in temperature measurement}

According to (6), the temperature at the point of scattering can be determined by measuring $R\left(T_{0}\right)$ and $R(T)$. Under actual conditions, however, there are several error factors as shown in Fig. 1. The temperature measurement accuracy may be affected by optical device characteristics such as the light source (wavelength), optical filter, photodiode, reference thermometer (uncertainty), optical switch, optical connector, splice, and sensing fiber.

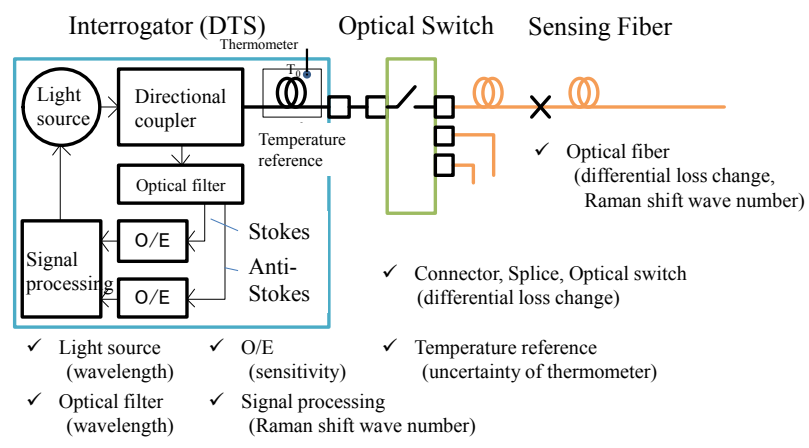

Fig. 1 Basic diagram of the DTS and error factors in temperature measurement.

Although a DTS vender can provide the individual correction data on each interrogator, the careful study and a calibration technique should be taken into consideration for highly accurate temperature measurement.

The next section describes 1) a method to find out the accurate Raman shift wave number of the sensing fiber (fiber having characteristics different from those of the built-in fiber is connected through the optical connecter, splice, or optical switch) and 2 ) the improved double-ended configuration insusceptible to the differential loss change in the sensing fiber and suitable for the field use.

\section{Novel calibration methods}

\subsection{Highly accurate measurement over the wide temperature range}

Let us set the sensing fiber (fiber having different characteristics from the built-in fiber) to be connected to the interrogator. The measured 
temperature $\left(T_{s}{ }^{\prime}\right)$ at the point of scattering can be calculated by measuring $R_{r}\left(T_{0}\right)$ and $R\left(T_{s}\right)$. Here, $T_{s}$ is the true temperature, and the Raman shift wave number of the sensing fiber is set to the same value as the built-in fiber. $R_{r}\left(T_{0}\right)$ and $R\left(T_{s}\right)$ are the ratios of anti-Stokes to Stokes intensities of the built-in fiber and the measured point, respectively.

$$
\begin{aligned}
& T_{s}{ }^{\prime}= \\
& \frac{h c \Delta v_{r}}{k} \cdot\left[\frac{1}{-\ln R\left(T_{s}\right)+\ln R_{r}\left(T_{0}\right)+\frac{h c \Delta v_{r}}{k} \cdot \frac{1}{T_{0}}}\right] .
\end{aligned}
$$

The actually measured $R\left(T_{s}\right)$ can be expressed by

$$
R\left(T_{s}\right)=D L_{\text {conn }} \cdot \frac{\left(v_{0}+\Delta v_{r}\right)^{4}}{\left(v_{0}-\Delta v_{r}\right)^{4}} \cdot \exp \left(-\frac{h c \Delta v_{s}}{k T_{s}}\right)
$$

where $D L_{\text {conn }}$ is the differential loss of the optical connecter, and $\Delta v_{s}$ is the Raman shift wave number of the sensing fiber. Then, $R_{r}\left(T_{0}\right)$ is given by

$$
R_{r}\left(T_{0}\right)=\frac{\left(v_{0}+\Delta v_{r}\right)^{4}}{\left(v_{0}-\Delta v_{r}\right)^{4}} \cdot \exp \left(-\frac{h c \Delta v_{r}}{k T_{0}}\right)
$$

where $\Delta v_{r}$ is the Raman shift wave number, and $T_{0}$ is the absolute temperature of the built-in fiber.

Using (7) (8) and (9), $T_{s}$ is given by $T_{s}=\frac{h c \Delta v_{s}}{k}$.

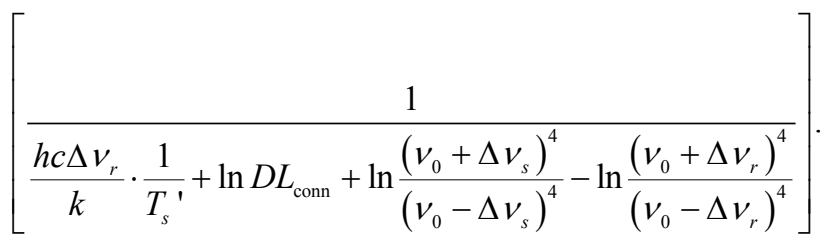

When the temperature is measured at the scattering point, the temperature calculated by (7) and the true temperature are set to $T_{1}{ }^{\prime}$ and $T_{1}$. Then, at another temperature measurement, the temperature calculated by (7) and the true temperature are set to $T_{2}{ }^{\prime}$ and $T_{2}$ as well. Then, $T_{s}$ and the true Raman shift wave number of the sensing fiber can be expressed by

$$
\begin{aligned}
& T_{s}=\Delta v_{s} \cdot \frac{1}{\frac{\Delta v_{r}}{T_{s}{ }^{\prime}}-\frac{\Delta v_{r}}{T_{1}{ }^{\prime}}+\frac{\Delta v_{s}}{T_{1}}} \\
& =\Delta v_{s} \cdot \frac{1}{\frac{\Delta v_{r}}{T_{s}{ }^{\prime}}-\frac{\Delta v_{r}}{T_{2}{ }^{\prime}}+\frac{\Delta v_{s}}{T_{2}}} \\
& \Delta v_{s}=\Delta v_{r} \cdot \frac{T_{1}{ }^{\prime}-T_{2}{ }^{\prime}}{T_{1}{ }^{\prime} \cdot T_{2}{ }^{\prime}} \cdot \frac{T_{1} \cdot T_{2}}{T_{1}-T_{2}} .
\end{aligned}
$$

Using $\Delta v_{s}$ given by (12), the temperature can be determined by (11) highly accurately [3]

On the basis of above examination, we evaluated the temperature measurement accuracy using the proposed calibration method. Firstly, we determined the true Raman shift wave number $\Delta v_{s}$ of the sensing fiber by using (12) at $+80{ }^{\circ} \mathrm{C}\left(T_{1}\right)$ and $+300{ }^{\circ} \mathrm{C}\left(T_{2}\right)$. The measured temperature difference between the DTS and the reference thermometer was within $+/-0.2^{\circ} \mathrm{C}$ in the wide temperature range from $+80{ }^{\circ} \mathrm{C}$ to $+300{ }^{\circ} \mathrm{C}$.

When $\Delta v_{s}$ was unknown, the temperature difference was more than $+/-1{ }^{\circ} \mathrm{C}$. Here, $\Delta v_{s}$ was set to the same value of $\Delta v_{r}$, and the DTS was calibrated at $+80{ }^{\circ} \mathrm{C}$ and $+300{ }^{\circ} \mathrm{C}$. The measured temperature was calculated using the linear approximation. Figure 2 compares the evaluated results with those of the conventional method.

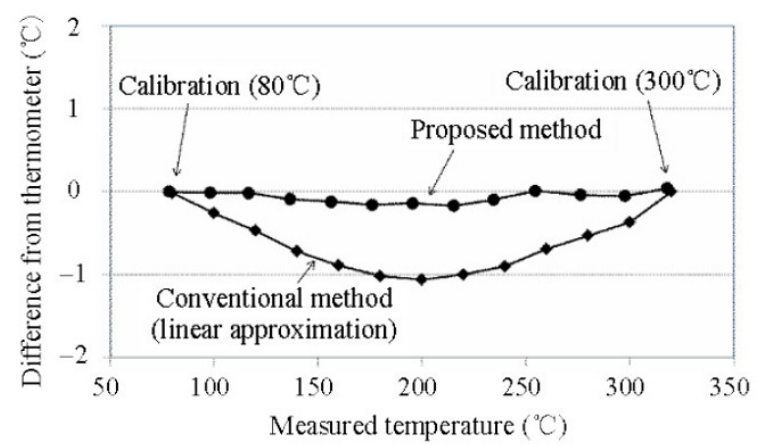

Fig. 2 Evaluated results using the proposed calibration method.

\subsection{Improved double-ended configuration insusceptible to the differential loss change}

To compensate for the differential loss, several methods have been proposed, by using two light 
sources with different wavelengths $[4,5]$ and most notably using a sensing fiber in a double-ended (loop) configuration (requiring the access to both fiber ends). The latter technique robustly and reliably compensates for the differential loss, through the calculation of the geometric mean associated to Stokes and anti-Stokes lights measured in both forward and backward directions. In this way, the effects of the fiber attenuation and local losses are inherently cancelled out in temperature estimation through the anti-Stokes/Stokes signal ratio, leading to auto-calibrated measurements that do not depend on optical fiber loss variations during the sensor lifetime.

This double-ended configuration requires installing the optical fiber for temperature reference outside the interrogator. However, this requirement is not acceptable from the viewpoint of convenience. To overcome this inconvenience, we propose a method that takes advantage of the double-ended configuration while utilizing the built-in temperature reference fiber [6].

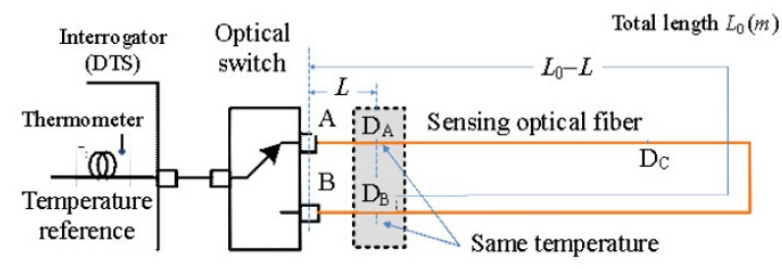

Fig. 3 Improved double-ended configuration.

In Fig. 3, the near fiber end $\mathrm{A}$ and far fiber end $\mathrm{B}$ are set on the same temperature condition. The anti-Stokes/Stokes signal ratio at the point $D_{A}$ measured at $\mathrm{A}$ is expressed as

$$
R_{\text {as } s t_{-} \mathrm{D}_{\mathrm{A}}}^{\prime}=R_{\text {as/st_ } \mathrm{D}_{\mathrm{A}}} \cdot D L_{\text {fiber }}\left(L_{1}\right)
$$

where $R_{\text {as st_ } \mathrm{D}_{\mathrm{A}}}$ is the anti-Stokes/Stokes signal ratio at the point $\mathrm{D}_{\mathrm{A}}, D L_{\text {fiber }}\left(L_{1}\right)$ is a differential loss, and $L_{1}$ is the length from $\mathrm{A}$ to $\mathrm{D}_{\mathrm{A}}$. Also, the anti-Stokes/Stokes signal ratio at the point $D_{B}$ measured at $\mathrm{A}$ is expressed as

$$
R_{\text {asst_ } \_\mathrm{D}_{\mathrm{B}}}^{\prime}=R_{\text {assst_ } \mathrm{D}_{\mathrm{B}}} \cdot D L_{\text {fiber }}\left(L_{0}-L_{1}\right)
$$

where $R_{\text {as st_ } D_{\mathrm{B}}}$ is the anti-Stokes/Stokes signal ratio at the point $\mathrm{D}_{\mathrm{B}}$. The temperatures at the points $\mathrm{D}_{\mathrm{A}}$ and $\mathrm{D}_{\mathrm{B}}$ are the same, and $D L_{\text {fiber }}\left(L_{1}\right) \cong 1$, $D L_{\text {fiber }}\left(L_{0}-L_{1}\right) \cong D L_{\text {fiber_total }}$. Then,

$$
\begin{aligned}
& \frac{R_{\text {as } / \mathrm{st} \_\mathrm{D}_{\mathrm{B}}}^{\prime}}{R_{\text {as st__ } \mathrm{D}_{\mathrm{A}}}^{\prime}}=\frac{R_{\text {as } / \mathrm{st} \_\mathrm{D}_{\mathrm{B}}}}{R_{\text {as } / \mathrm{st} \_\mathrm{D}_{\mathrm{A}}}} \cdot \frac{D L_{\text {fiber }}\left(L_{0}-L_{1}\right)}{D L_{\text {fiber }}\left(L_{1}\right)} . \\
& =D L_{\text {fiber_total }}
\end{aligned}
$$

The combined signal (geometric mean) of the anti-Stokes/Stokes signal ratios at the point $D_{C}$ measured at $\mathrm{A}$ and measured at $\mathrm{B}$ is expressed as

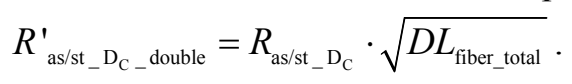

By using (15) and (16), the signals of the built-in (single-ended) and sensing (double-ended) fibers can be combined.

On the basis of the above discussion, we experimented with the temperature measurement using the sensing fiber in which the differential loss change occurred.

Figure 4 shows the experimental results of the measurement over the section with the constant temperature $\left(+24.8 \quad{ }^{\circ} \mathrm{C}\right)$. The temperature measurement using the improved double-ended configuration is not affected by the differential loss change in the sensing fiber although the temperature measurement using the conventional single-ended configuration is strongly affected. Also, Fig. 4 shows the normal double-ended configuration without correction using $D L_{\text {fiber_total }}$ still has some errors.

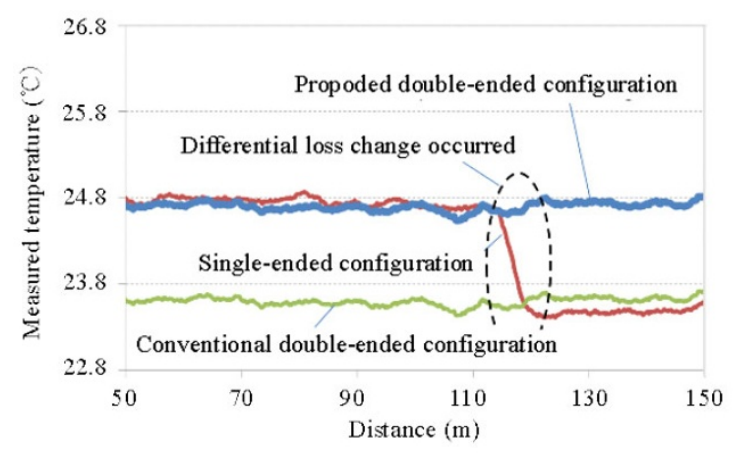

Fig. 4 Test results of improved double-ended configuration.

\section{Environmental robustness $[7,8]$}

A wide operating temperature range between $-45^{\circ} \mathrm{C}$ and $+70{ }^{\circ} \mathrm{C}$ can be achieved by using the temperature control of the laser diode (LD), which 
can be controlled even if the ambient temperature significantly changes. This is because the elements to be temperature controlled have been assembled as compactly as possible. Also, to keep the multiplication factor of the avalanche photo diode (APD) constant regardless of its ambient temperature, the bias voltage is controlled while the temperature of its vicinity is monitored.

For explosion proofing, the interrogator has achieved the explosion proof construction as the energy limited apparatus by suppressing electric power at connecting points of the electronic circuits. To achieve resistance to corrosion, the parts have been selected by using accelerated corrosion tests. Figure 5 shows the results of the evaluation test.

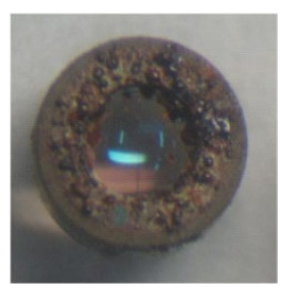

Sample (a)

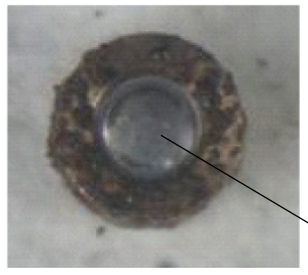

Sample (b)
Fig. 5 Results of accelerated corrosion test for optical lenses.

Commercially available optical lenses were evaluated for 60 days in the high temperature and high humidity atmosphere containing hydrogen sulfide, which was equivalent to a 10 -year test in a corrosive gas environment. The lens of Sample (b) became clouded due to improper coating materials, making it unusable. On the other hand, Sample (a) indicated no changes in the optical performance, although some rust on the metal part of the lens housing was found. As a result, the interrogator complies with some standards for explosion proofing and resistance to corrosive gases.

Figure 6 shows temperature measurement in a harsh environment, where the ambient temperature of the interrogator is changed stepwise from $+70^{\circ} \mathrm{C}$ to $-45{ }^{\circ} \mathrm{C}$ to evaluate the dependency of its measurement on the ambient temperature. During the process of changing the ambient temperature of the interrogator while keeping the optical fiber to be measured at the normal temperature, the fluctuation of the temperature measurement values does not exceed $+/-1{ }^{\circ} \mathrm{C}$, including the room temperature fluctuation of the optical fiber.

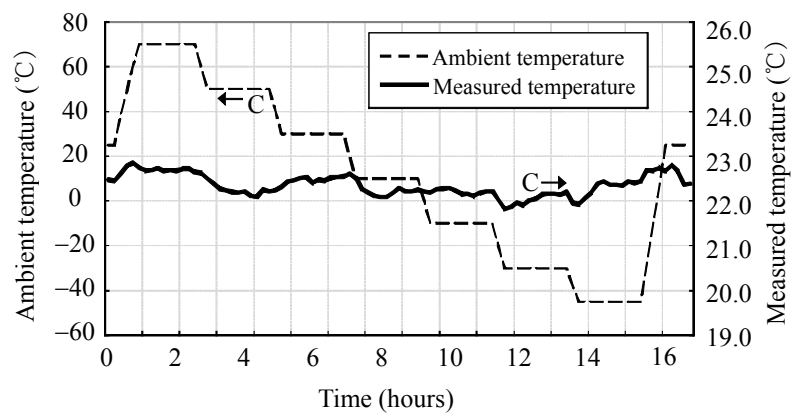

Fig. 6 Example of characteristics of dependency on ambient temperature.

Figure 7 shows an application example of the DTS at a resource mining site. The infrastructure for the power or communications is often not developed at resource mining sites. In an example for an outdoor panel solution, the power is supplied with solar panels and rechargeable batteries, and wireless modems are used for communications.

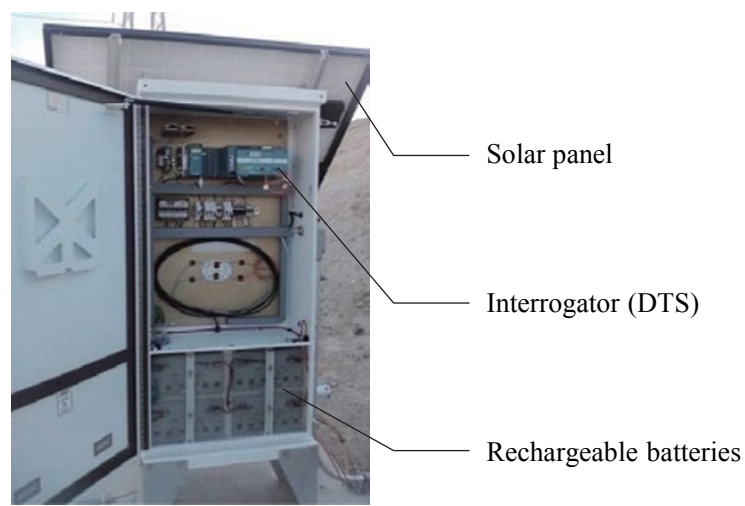

Fig. 7 Application example of the DTS at a resource mining site.

\section{Summary}

In summary,

(1) We proposed a novel temperature calibration method. By using this method, the temperature was measured accurately within $+/-1{ }^{\circ} \mathrm{C}$ in a wide temperature range from $+80^{\circ} \mathrm{C}$ to $+300^{\circ} \mathrm{C}$.

(2) The simple and fully automatic correction 
technique was presented that used the improved double-ended configuration with the built-in fiber (for temperature reference).

(3) We achieved high tolerability in harsh environments and operation with the limited power supply, such as that from solar panels and rechargeable batteries. The infrastructure cost (doghouse, power supply, air-conditioning, and maintenance support) can be significantly reduced.

(4) By using the developed robust DTS, the temperature measurement fluctuated within $+/-1{ }^{\circ} \mathrm{C}$ in the wide operation temperature range from $-45^{\circ} \mathrm{C}$ to $+70^{\circ} \mathrm{C}$.

Open Access This article is distributed under the terms of the Creative Commons Attribution License which permits any use, distribution, and reproduction in any medium, provided the original author(s) and source are credited.

\section{References}

[1] A. H. Hartog, "Raman sensors and their applications," in Proc. SPIE (22nd International Conference on Optical Fiber Sensors), vol. 8421, pp.
84210K-1-84210K-6, 2012.

[2] J. P. Dakin, D. J. Pratt, G. W. Bibby, and J. N. Ross, "Distributed optical fiber Raman temperature sensor using a semiconductor light source and detector," Electronics Letters, vol. 21, no. 13, pp. 569-570, 1985.

[3] T. Fukuzawa, H. Shida, K. Oishi, H. Agawa, and S. Adachi, "Novel temperature calibration method for Raman distributed temperature sensor (DTS)," in Proc. 50th Meeting on Lightwave Sensing Technology, LST50-18, pp. 117-122, 2012 (in Japanese).

[4] C. E. Lee, "Self-calibrating technique enables long-distance temperature sensing," Laser Focus World, vol. 43, no. 8, pp. 101-117, 2007.

[5] K. Suh and C. Lee, "Auto-correction method for differential attenuation in a fiber-optic distributed-temperature sensor," Optics Letters, vol. 33, no. 16, pp. 1845-1847, 2008.

[6] T. Fukuzawa, H. Shida, K. Oishi, H. Agawa, and N. Takeuchi, "Automatic correction technique for Raman distributed temperature sensor (DTS) using improved double-ended configuration," in Proc. IEEJ Technical Meeting on Optical Application and Vision, LAV-13-012, 2013 (in Japanese).

[7] N. Takeuchi and T. Fukuzawa, "Distributed temperature sensor (DTS) for use in hash environments," in Proc. IEEJ Technical Meeting on Optical Application and Vision, LAV-12, 2012 (in Japanese).

[8] Y. E. Corporation, "DTSX200 distributed temperature sensor," Journal of the Society of Instrument and Control Engineers, vol. 51, no. 3, pp. 310-311, 2012 (in Japanese). 